
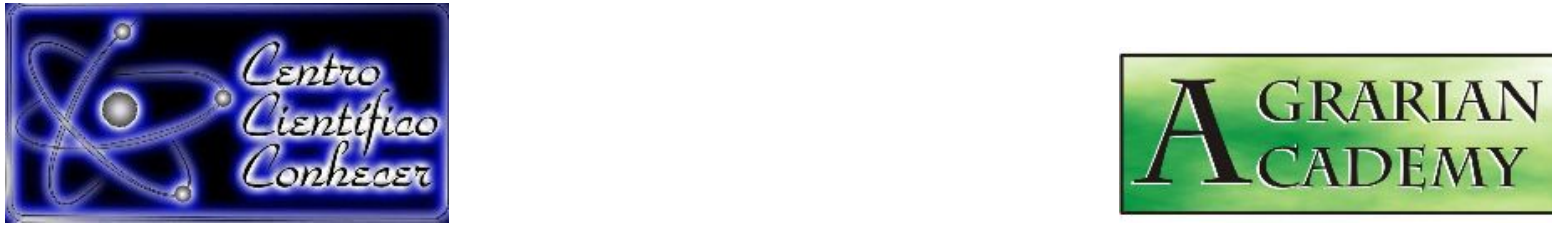

\title{
INDICADORES DE SUSTENTABILIDADE COM BASE NA QUALIDADE DO SOLO E ACÚMULO DE FITOMASSA EM PASTAGENS DEGRADADAS
}

\footnotetext{
Vicente José Laamon Pinto Simões ${ }^{1}$; Maurício Luiz de Mello Vieira Leite ${ }^{2}$; Eduardo Soares de Souza ${ }^{2}$; Leandro Ricardo Rodrigues de Lucena ${ }^{3}$; José Lypson Pinto Simões Izidro ${ }^{1}$

${ }^{1}$ Discente de Pós-Graduação em Produção Vegetal, Universidade Federal Rural de Pernambuco, Serra Talhada, PE, Brasil, (laamoneng.agro@gmail.com); ${ }^{2}$ Prof. Dr. Departamento de Agronomia, Universidade Federal Rural de Pernambuco, Serra Talhada, PE, Brasil;

${ }^{3}$ Prof. Dr. Departamento de Zootecnia, Universidade Federal Rural de Pernambuco, Serra Talhada, PE, Brasil.
}

Recebido em: 14/07/2018 - Aprovado em: 28/07/2018 - Publicado em: 31/07/2018 DOI: 10.18677/Agrarian_Academy_2018a26

\begin{abstract}
RESUMO
O principal interesse deste estudo é auxiliar na compreensão do processo evolutivo de degradação das pastagens e a importância da determinação da sustentabilidade dessas áreas a partir de indicadores estabelecidos com base na qualidade do solo e acúmulo de fitomassa, visando fornecer subsídios para a adoção de práticas de manejo que permitam incrementar o rendimento das culturas, garantindo a contínua sustentabilidade e conservação dos ecossistemas. Um indicador de sustentabilidade é um instrumento essencial para obtenção de informações sobre uma dada realidade, principalmente, para evidenciar modificações que ocorrem no ambiente devido à ação antrópica. É impossível determinar a sustentabilidade de um sistema considerando apenas um indicador, ou indicadores que se refiram a apenas um aspecto do sistema. Nesse sentido, deve-se empregar sempre um conjunto de indicadores para avalição da sustentabilidade, pois somente através das relações entre eles é possível estabelecer um padrão de respostas dos sistemas e então prever futuras condições. Diante da variedade de indicadores de sustentabilidade associados à qualidade do solo, há também índices estabelecidos com base no crescimento e produção das espécies forrageiras nas áreas de pastagens. Desse modo, estudos sobre a utilização de indicadores de sustentabilidade estabelecidos com base na qualidade do solo e acúmulo de fitomassa, tornam-se fundamentais para dar subsidio ao planejamento dos sistemas produtivos em diferentes regiões e garantir a contínua sustentabilidade e conservação desses ecossistemas.
\end{abstract}

PALAVRAS-CHAVE: degradação de pastagens; sistemas agroflorestais; qualidade do solo. 


\title{
SUSTAINABILITY INDICATORS BASED ON THE QUALITY OF SOIL AND PHYTOMASS ACULVULATION IN DEGRADED PASTURES
}

\begin{abstract}
The main interest of this study is to assist in understanding the evolutionary process of pasture degradation and the importance of determining the sustainability of these areas based on indicators established based on soil quality and phytomass accumulation, aiming to provide subsidies for the adoption of management to increase crop yields, ensuring the continued sustainability and conservation of ecosystems. An indicator of sustainability is an essential tool for obtaining information about a given reality, mainly to show changes that occur in the environment due to anthropic action. It is impossible to determine the sustainability of a system by considering only one indicator, or indicators that refer to only one aspect of the system. In this sense, a set of indicators should always be used to evaluate sustainability, since only through the relationships between them can it be possible to establish a pattern of system responses and then predict future conditions. Given the variety of sustainability indicators associated with soil quality, there are also established indices based on the growth and production of forage species in pasture areas. Thus, studies on the use of sustainability indicators established on the basis of soil quality and phytomass accumulation, become fundamental to subsidize the planning of production systems in different regions and ensure the continued sustainability and conservation of these ecosystems.
\end{abstract}

KEYWORDS: pasture degradation; agroforestry systems; soil quality.

\section{INTRODUÇÃO}

As análises referentes ao setor do agronegócio, no contexto mundial, apontam que a maioria dos países está priorizando o desenvolvimento da pecuária baseada exclusivamente em pastos (KOTLYAROVA et al., 2013). No entanto, à medida que a pressão econômica está se tornando mais forte, as práticas inadequadas de manejo, como por exemplo, o pastejo excessivo, a violação da sazonalidade da oferta de forragem e os investimentos mínimos ou inexistentes no desenvolvimento sustentável das pastagens levam a degradações severas dessas áreas (ANGASSA; OBA, 2010; SATTLER et al., 2018).

Atualmente, a maior parte dos ecossistemas de pastagens está seriamente degradada, várias espécies forrageiras com excelente valor nutricional desapareceram ou se tornaram raras, e os solos foram ou estão sendo severamente esgotados (ANGASSA; OBA, 2010; SMIRNOVA et al., 2017). Em decorrência disso, segundo Carvalho et al. (2017), é crescente a busca por gramíneas pouco exigentes em termos nutricionais, tolerantes às condições adversas de cultivo e de alta produtividade, visando substituir aquelas que foram utilizadas sem, no entanto, preocupar-se com os problemas que ocasionaram à queda da produtividade da pastagem. Isso terá implicações não apenas na estabilidade climo-edáficos e ecológicos e na resiliência dessas áreas, mas trará sérias consequências para a pecuária, que em algumas regiões poderá tornar-se uma atividade economicamente inviável e, em longo prazo, acelerar o êxodo rural.

A degradação das áreas de pastagens é um fenômeno global, definido como um processo evolutivo de perda de vigor da espécie forrageira, ao ponto de torna-se incapaz de uma recuperação natural e sem a mínima possibilidade de sustentar os níveis de produção e qualidade exigida pelos animais, bem como de superar os 
efeitos nocivos de pragas, doenças e plantas invasoras (CARVALHO et al., 2017) ou como um processo em que a produção da forragem diminui simultaneamente com a sua qualidade, mesmo que em épocas adequadas ao crescimento (SOARES FILHO et al., 1992).

Em virtude dessa insegurança produtiva, relacionada com as áreas de pastagens degradadas, é notória a progressiva conscientização com relação à preservação dos recursos naturais, sobretudo através de práticas sustentáveis no manejo agrícola segundo Theodoro et al. (2011). No entanto, de acordo com diversos autores (VERONA et al., 2007; CÂNDIDO et al., 2015; GUIMARÃES et al. 2015) é fundamental a avaliação da sustentabilidade dos diferentes sistemas de produção, por meio de indicadores que permitem mensurar os pontos positivos ou negativos de determinado ecossistema, de modo a auxiliar na condução de um manejo de forma sustentável.

Desse modo, o principal interesse deste estudo é auxiliar na compreensão do processo evolutivo de degradação das pastagens e a importância da determinação da sustentabilidade dessas áreas a partir de indicadores estabelecidos com base na qualidade do solo e acúmulo de fitomassa, visando fornecer subsídios para a adoção de práticas de manejo que permitam incrementar o rendimento das culturas, garantindo a contínua sustentabilidade e conservação dos ecossistemas.

\section{Degradação agrícola e biológica das áreas de pastagens}

De acordo com Dias-Filho (2011) a caracterização de uma pastagem como degradada ou em degradação pode variar dentro de uma faixa relativamente ampla de condições. Ainda, segundo esse autor, os extremos dessas condições são denominados de degradação agrícola e biológica. Na primeira circunstância, ocorre uma mudança na composição botânica do ecossistema, devido à sobreposição das plantas daninhas sobre a gramínea forrageira, o que ocasiona temporariamente em uma drástica redução da produtividade de fitomassa da pastagem, em virtude da pressão competitiva praticada entre as espécies, causando, portanto, uma diminuição significativa da capacidade de suporte dessas áreas. Nessa situação, segundo Dias-Filho (2007), em certos casos, não ocorre à deterioração das propriedades físico-químicas do solo, pode-se até melhorar as características do mesmo, através do aumento da cobertura vegetal, proporcionada pelas espécies invasoras.

A degradação agrícola é agravada por vários fatores que agem individualmente ou em conjunto, como o preparo incorreto do solo, uso de espécies forrageiras pouco adaptadas às condições edafoclimáticas da área de cultivo, utilização de sementes com baixo vigor, que por sua vez, ocasiona em falhas iniciais no estande e perda da produtividade e, principalmente, em razão da negligência na reposição dos nutrientes esgotados do solo no processo produtivo, erosão, lixiviação e volatilização (DIAS- FILHO, 2011; CARVALHO et al., 2017). Além desses fatores, de acordo com Sattler et al. (2018), as mudanças climáticas provocam condições ambientais que podem acentuar o processo de degradação.

Segundo Dias-Filho (2011), as regiões sob floresta no trópico úmido, como por exemplo, a Amazônia Continental, onde os períodos de seca não são tão severos, a degradação agrícola é geralmente a forma mais frequente de degradação. No entanto, em locais com período seco prolongado e com características edafoclimáticas típicas dos ecossistemas de Cerrado e Caatinga, a degradação de pastagem mais comum é a biológica (DIAS-FILHO, 2011). 
Dereczynski et al. (2013) e Salazar et al. (2015), conforme modelos climáticos que realizam projeções do clima futuro, descreveram que os eventos de secas podem afetar com maior frequência e intensidade algumas regiões do Brasil, como por exemplo o sudeste, ao mesmo tempo em que as precipitações pluviais serão mais intensas. Dessa forma, origina-se um cenário favorável para degradação biológica na maioria das áreas de pastagens do Brasil, o que pode representar um forte entrave para atividade da pecuária no futuro.

$\mathrm{Na}$ degradação biológica, as práticas inadequadas de manejo da terra afetam diretamente as características físico-químicas do solo, ocasionando redução acentuada da capacidade de sustentar a produção vegetal na área e, como consequência, diminui significativamente a produtividade dos pastos e, no pior dos casos, torna as terras inapropriadas para serem usadas para agricultura e pastagem de maneira economicamente viável. Nessas circunstâncias, a degradação é mais drástica e torna-se muito mais complexa e cara a recuperação e restauração dessas áreas, devido à intensa redução da qualidade do solo, a espécie forrageira é gradativamente substituída por plantas de baixa produtividade e pouco exigentes em fertilidade do solo ou, em alguns casos, ocorrem a total remoção da vegetação da área, resultando em solos descobertos e altamente susceptíveis aos processos de erosão (DIAS-FILHO, 2014).

Pastagens degradadas não são resistentes à erosão, e o desgaste das rochas e dos solos resultantes desse processo, pode gerar inúmeros problemas sociais, econômicos e ambientais, tais como o fenômeno da desertificação, que é considerado um problema de dimensões globais, comprometendo, principalmente, as regiões de clima árido, semiárido e subúmido seco da Terra (SILVA et al., 2018; SOUSA et al., 2018). As forças naturais da erosão hídrica combinadas com a densidade excessiva do gado e a falta de manejo da pastagem estão agravando o risco de degradação (SILVA; BOTELHO 2014; GALDINO et al. 2016). De acordo com Alexandridis et al. (2013), a erosão do solo é um dos principais motivos que levam à perda de produtividade nas gramíneas forrageiras em áreas degradadas, tornando-se um problema grave em todo o mundo.

A perda de solo devido aos processos erosivos pode provocar queda drástica na qualidade física do solo e resultar em uma série de problemas ambientais, como, por exemplo, a sedimentação e elevada turbidez da água (ATUCHA et al., 2013; FORTIN et al., 2015). De acordo com Silva e Botelho (2014), o subsolo exposto por esses processos, com teores de argila normalmente acima de $40 \mathrm{~g} \mathrm{~kg}^{-1}$ e uma densidade acima de $1,6 \mathrm{~g} \mathrm{~cm}^{-3}$, contribuem ainda mais para 0 escoamento superficial, o que acelera consideravelmente a erosão e perda de nutrientes do solo. Diversos estudos evidenciaram que as perdas de nutrientes podem causar redução da fertilidade do solo e a eutrofização dos estuários (PIMENTEL et al., 1995; JARVIE et al., 2013). Segundo Aguiar et al. (2010) e Oliveira et al. (2013), devido ao escoamento, o carbono orgânico e nutrientes valiosos, como fósforo, potássio, cálcio e magnésio são perdidos junto com o solo e a água. Dessa forma, um ciclo vicioso é iniciado, resultando no declínio da qualidade física e empobrecimento da fertilidade dos solos.

No Brasil, assim como em outros países no mundo, as pesquisas realizadas sobre o processo de erosão, são restritas a áreas agrícolas e voltadas para o desenvolvimento de práticas de manejo do solo que diminuam os impactos causados por esse problema (ROCHA-JUNIOR et al., 2017). No entanto, há poucos estudos para avaliar os impactos da erosão nas perdas de solo e água em áreas de pastagens (TEAGUE et al., 2010; JEMAI et al., 2013; SHAKESBY et al., 2013).

AGRARIAN ACADEMY, Centro Científico Conhecer - Goiânia, v.5, n.9; p.256 2018 
De acordo com diversos autores (BERTOL et al., 2010; DEFERSHA et al., 2010; OLIVEIRA et al., 2013) para um planejamento de conservação do solo, é de extrema importância o conhecimento sobre como as diversas práticas de manejo influenciam as taxas de perda do solo. Nesse sentido, torna-se necessário o desenvolvimento de pesquisas com o intuito de avaliar práticas de manejo de pastagens que venham a contribuir com conhecimentos sobre o impacto dos processos erosivos na qualidade do solo.

Segundo Sattler et al. (2018), à pressão exercida no solo devido ao acréscimo no número de animais por unidade de área, provoca o aumento da densidade aparente e, consequente redução da porosidade e infiltração de água. Tais afirmações corroboram com Tormena et al. (2007) e Moreira et al. (2014) quando descreveram que modificações na estrutura do solo ocasionam mudanças na arquitetura do espaço poroso disponível para aeração, armazenamento de água e desenvolvimento radicular, além de provocar mudanças na resistência do solo à penetração das raízes. Além disso, conforme Ren et al. (2017) e Abdalla et al. (2018) o pastejo dos animais influencia nas características fenotípicas das gramíneas forrageiras, comprometendo a fotossíntese foliar, a respiração e o completo desenvolvimento das plantas, também pode apresentar influência indireta sobre as espécies forrageiras predominantes no ecossistema, alterando dessa forma o microambiente do solo.

Todos esses processos negativos resultam no esgotamento da biodiversidade, diminuem a produtividade dos ecossistemas de pastagens e, consequentemente, levam à deterioração dos recursos forrageiros (SQUIRES, 2012). Assim sendo, de acordo com Passos et al. (2017), o uso eficiente da água se tornará cada vez mais importante, visto o cenário projetado para a década atual, onde a crise da água representará um problema em diversas regiões. Dessa forma, os mesmos autores ressaltaram que a gestão eficiente do solo, dadas as particularidades de cada região do país, é fundamental para sustentabilidade na agricultura, considerando que, o solo desempenha papel importante no armazenamento de água e, dessa forma, faz parte do ciclo hidrológico.

\section{Tecnologias e práticas de manejo em pastagens degradadas}

No Brasil, bem como em diversos países do mundo, há uma tendência de produção pecuária baseada quase que no uso exclusivo de pastagens utilizadas sob pastejo para as próximas décadas. De acordo com Sattler et al. (2018), sem uma intervenção em busca do desenvolvimento sustentável e incentivos para o abandono de pastagens íngremes, a degradação contínua dessas áreas levará à escassez de pastagens adequadas. Conforme esses autores, no Brasil é crescente a conscientização com relação à importância e necessidade de conservação da biodiversidade. Segundo Strassburg et al. (2014) através do manejo adequado e com base na sustentabilidade do sistema de produção, a produtividade das pastagens brasileiras pode aumentar para mais de $50 \%$ até o ano de 2040 , sem necessidade de converter habitats naturais em ambientes cultivados. Nesse sentido, cientistas do mundo todo estão desenvolvendo pesquisas sobre a restauração de pastagens degradadas ou em degradação, e estudando medidas para 0 manejo sustentável dos recursos da terra (HAN et al., 2008; HOU et al., 2008).

A possibilidade de diversificação produtiva em uma mesma unidade de manejo e o aumento de renda através da implantação de sistemas agrosilvipastoris tem ganhado espaço e feito muito sucesso, pois a integração de dois ou mais 
sistemas de produção proporciona maiores rendimentos e maior flexibilidade na produção (BALBINO et al., 2011; REGO et al., 2017). Os ecossistemas de pastagens possuem elevado potencial para o sequestro de carbono, além disso, promovem o controle da erosão, conservação dos recursos hídricos e de solo e, dessa forma, favorecem a preservação da biodiversidade (REGO et al., 2017).

De acordo com Laliberté et al. (2012) e Odriozola et al. (2017), as interações planta-solo são de extrema importância na manutenção da composição da comunidade vegetal, além disso, as propriedades do solo exercem um papel fundamental na transição de pastagens degradadas para ecossistemas restaurados. Han et al. (2008) e Jing et al. (2014) ressaltaram que a exclusão de pastejo tornouse uma prática primária indispensável para restauração de pastagens degradadas, principalmente, em regiões semiáridas. A reabilitação de pastagens através de tecnologias da bioengenharia para controlar os processos erosivos, em conjunto com práticas de manejo como o pastejo rotacionado, podem interromper ou pelo menos diminuir consideravelmente a degradação das pastagens (SATTLER et al., 2018). Essa afirmação corrobora com Oliveira et al. (2017) ao destacarem que as novas tecnologias de produção agropecuária são de extrema importância para o desenvolvimento de sistemas mais sustentáveis, uma vez que, reduzem os impactos negativos nas propriedades do solo e na microbiota benéfica associada as plantas.

\section{Indicadores de sustentabilidade}

Em várias áreas da ciência, o conceito da "sustentabilidade" é extremamente discutido e sempre relacionado com a conservação dos recursos naturais, associada, com o desenvolvimento econômico e social. Conforme Sant'anna et al. (2017), considera-se um sistema ou processo como sustentável quando o mesmo é realizado de forma orientada para o benefício da população sem agredir o meio ambiente. No entanto, vários autores (CANELLAS et al., 2003; RANGEL ; SILVA, 2007; BORLACHENCO; GONÇALVES, 2017), destacaram que a implantação de atividades agropecuárias sem as devidas preocupações com a sustentabilidade, vem promovendo desequilíbrio nos ecossistemas como, por exemplo, a utilização dos solos até o esgotamento e a degradação, tornando-os impróprios para explorações futuras.

Existe uma pressão social relacionada com a preservação ambiental, devido aos impactos já observados pela escassez dos recursos naturais e aumento da poluição (KUZMA et al., 2015). Em um sistema de produção, as necessidades devem ser supridas sem intervir nas possibilidades futuras, ou seja, os recursos indispensáveis para a produção no futuro não devem ser esgotados para atender as necessidades atuais (GARCEZ, 2014). Dessa forma, torna-se evidente a necessidade de quantificar os impactos dos diferentes tipos de manejo, através de indicadores de sustentabilidade que possam guiar a ação e subsidiar o desenvolvimento sustentável, obedecendo a parâmetros que assegurem a manutenção dos recursos naturais no futuro.

Um indicador de sustentabilidade é um instrumento essencial para obtenção de informações sobre uma dada realidade, principalmente, para evidenciar modificações que ocorrem no ambiente devido à ação antrópica (CÂNDIDO et al., 2015; GUIMARÃES et al., 2015). É impossível determinar a sustentabilidade de um sistema considerando apenas um indicador, ou indicadores que se refiram a apenas um aspecto do sistema (MUKHERJEE; LAL, 2014). Nesse sentido, deve-se empregar sempre um conjunto de indicadores para avalição da sustentabilidade, 
pois somente através das relações entre eles é possível estabelecer um padrão de respostas dos sistemas e então prever futuras condições.

\section{Indicadores de sustentabilidade estabelecidos com base na qualidade do solo e acúmulo de fitomassa em áreas de pastagens}

Segundo Odum e Barrett (2011) o solo é o núcleo principal da organização dos ecossistemas, sejam eles terrestres ou de áreas úmidas, e um bom indicador da qualidade ambiental é a qualidade do solo, ou seja, quando há uma conservação das características que configuram a qualidade do solo, as ações antrópicas ou naturais ocorridas na paisagem são consideradas sustentáveis. A avaliação da qualidade do solo possibilita a determinação de medidas adequadas de manejo visando à conservação do solo e melhoras nos rendimentos das culturas (MARZAIOLI et al., 2010).

Veum et al. (2017) ressaltaram que a avaliação da qualidade do solo tem como objetivo determinar o desempenho das funções biológicas, químicas e físicas do solo e comparar com o seu potencial intrínseco. Assim sendo, de acordo com Budak et al. (2018), análises referentes ao estado atual da qualidade do solo, podem indicar com alta precisão a suscetibilidade da área aos processos de degradação, representando dessa forma uma importante ferramenta para conservação dos recursos naturais.

A caracterização das propriedades do solo, através da determinação de um conjunto mínimo de dados, possibilita estimar com exatidão a qualidade do solo (BUDAK et al., 2018). A degradação das características físicas do solo é um dos principais processos responsáveis pela redução da qualidade, tornando-se imprescindível o monitoramento dessas propriedades para o desenvolvimento de sistemas sustentáveis (BERTOL et al., 2001; SILVA et al., 2005). A utilização combinada de indicadores de qualidade física do solo derivados de medições diretas das propriedades hidráulicas, levam a informações consistentes e indispensáveis para detectar a degradação do solo e avaliar o efeito das mudanças no uso da terra (CASTELLINI et al., 2016). Ainda, conforme esses autores, os indicadores medidos e estimados mostraram unanimemente degradação severa da qualidade física do solo associada à compactação do solo, perda de matéria orgânica, diminuição do volume de macroporos e da capacidade de aeração do solo.

Algumas pesquisas (GONZALEZ-SOSA et al., 2010; AGNESE et al., 2011) relataram que o desmatamento pode afetar negativamente a qualidade física dos solos, devido a redução do teor de matéria orgânica, da porosidade e da condutividade hidráulica, bem como do aumento da densidade do solo. Essas alterações são evidentes sinais de degradação, que por sua vez, é agravada pela compactação do solo e, consequente, pela redução da circulação de ar e água, ocasionando em aumentos significativos de escoamento superficial e erosões (CERDÀ et al., 2010).

Os indicadores físicos mais utilizados são a densidade, a porosidade, a estabilidade de agregados, a resistência mecânica do solo à penetração e a infiltração (LIMA et al., 2007; ALVES et al., 2007). A densidade do solo é afetada por práticas de manejo que modificam a estrutura do solo e, por consequência, o arranjo e o volume dos poros. Essas modificações afetam as propriedades físico-hídricas do solo, diminuindo a disponibilidade de água para as plantas e aumentando a resistência do solo a penetração (SOLDÁ, 2012). Segundo Reichert et al. (2009), o 
manejo inadequado das pastagens, em conjunto com um intenso revolvimento do solo afetam o arranjo das partículas, reduzindo a porosidade e a infiltração de água.

A principal causa de compactação do solo em áreas de pastagens é o pisoteio animal (SATTLER et al., 2018). Cardoso et al. (2011) em estudo sobre a qualidade química e física do solo sob vegetação arbórea nativa e pastagens no Pantanal sul-mato-grossense, constataram que o pastejo contínuo na pastagem nativa resultou na degradação da qualidade física do solo, confirmada pela redução da porosidade total, macroporosidade e condutividade hidráulica saturada. A compactação dos solos reduz a atividade biológica e, devido ao aumento na densidade, pode limitar a permeabilidade e a disponibilidade de água e nutrientes para as plantas (FREDDI et al., 2007; JIMENEZ et al., 2008).

De acordo com Castellini et al. (2016), a capacidade do solo em armazenar e distribuir água é dada em função da curva de retenção de água e da condutividade hidráulica. Os impactos das práticas de manejo nas propriedades hidráulicas do solo podem ser avaliados através da determinação dos indicadores de qualidade física do solo relacionados com a retenção de água e condutividade hidráulica (REYNOLDS et al., 2009). A partir da curva de retenção de água do solo são obtidos indicadores como, por exemplo, a macroporosidade, a capacidade de água disponível e a capacidade relativa de campo, os quais possuem uma importância significativa na avaliação das práticas de uso da terra que representam o melhor manejo (REYNOLDS et al., 2002; REYNOLDS et al., 2008).

Segundo Freitas et al. (2017), conhecer as diferenças químicas e físicas do solo, ocasionadas pelas diferentes formas de manejo, possibilita o planejamento que envolva práticas capazes de melhorar o rendimento das culturas, sem a degradação dos ecossistemas. Essas afirmações corroboram com Cardoso et al. (2011), ao relatarem que avaliações das propriedades do solo são de importância considerável no monitoramento da conservação ambiental, pois permite caracterizar a situação atual e alertar sobre situações de risco. Desse modo, ressalta-se a importância da avaliação das propriedades físicas e químicas do solo como, por exemplo, a fertilidade, a densidade, a porosidade, a resistência do solo à penetração e a distribuição dos agregados, quando se pretende determinar os impactos dos diferentes sistemas de manejo e então realizar um planejamento adequado visando um desenvolvimento sustentável.

São vários os indicadores de qualidade do solo e sustentabilidade agrícola, dentre os vários já citados, pode-se destacar ainda como atributos dinâmicos do solo a matéria orgânica, a estabilidade dos agregados e a respiração total do solo, uma vez que apresentam alta sensibilidade às praticas de manejo e mudanças no uso da terra (MASTO et al., 2008; VALENTINI et al., 2015). Conforme vários autores (LAURINDO et al., 2009; RALISCH et al., 2010; PINHEIRO MACHADO, 2010; VALENTINI et al., 2015) a matéria orgânica desempenha um papel de extrema importância para manutenção de uma boa estrutura e, consequente, qualidade do solo, uma vez que atua como agente de formação e estabilização dos agregados. Dessa forma, a implantação de sistemas de manejo que permitam a proteção do solo pelo continuo aporte de resíduos orgânicos como, por exemplo, a serapilheira, torna-se uma excelente alternativa para assegurar a conservação das propriedades que caracterizam a qualidade dos solos.

De acordo com Parkin et al. (1996), a respiração do solo pode ser definida como sendo a produção de $\mathrm{CO}_{2}$ pelos processos metabólicos de organismos vivos do solo. Segundo Silva et al. (2010) as práticas que favorecem a adição, ou ocasionam na remoção de material vegetal do solo, afetam diretamente a biomassa 
microbiana, sendo essas possivelmente avaliadas pelo quantitativo de gás carbônico produzido. Paustian et al. (2000) afirmaram que as perturbações físicas no solo podem promover a emissão de $\mathrm{CO}_{2}$ na atmosfera. Dessa forma, torna-se notório que o processo da respiração do solo pode ser intensificado ou amenizado por diversos fatores, sendo assim considerado um indicador extremamente sensível às alterações do ambiente, capaz de evidenciar modificações e quantificar os impactos dos diferentes tipos de manejo.

Valentini et al. (2015) relataram que a velocidade dessa liberação de carbono vai depender de diversos fatores como, por exemplo, microrganismos, umidade e temperatura do solo e qualquer outro fator que afete as condições microclimáticas do solo e sua interface com a atmosfera podem influenciar na taxa de respiração. De acordo com Davidson et al. (2002) a respiração do solo pode ser medida com equipamentos de infravermelho (IRGAs) em câmaras fechadas, ou ainda por métodos baseados na absorção de $\mathrm{CO}_{2}$ através de uma solução álcali.

Para avaliar a qualidade físico-hídrica do solo podem ser utilizados oito indicadores selecionados da literatura entre os quais são fornecidas faixas ótimas ou limites críticos. Os indicadores são: densidade do solo (DS), teor de carbono orgânico (OC), índice de estabilidade estrutural (IES), condutividade hidráulica saturada (Ks), macroporosidade (Pmac), capacidade de ar (CA), capacidade de água disponível para planta (CADP) e a capacidade de campo relativa (CCR) (PIERI, 1992; TOPP et al., 1997; REYNOLDS et al., 2007; 2009). A literatura atual (AGNESE et al., 2011; BAGARELLO et al., 2011; KELISHADI et al., 2014; OZALP et al., 2015; IOVINO et al., 2016) registra estudos ressaltando que os indicadores selecionados foram utilizados com sucesso para fornecer avaliações da capacidade do solo em armazenar e fornecer ar e água para as raízes, bem como, evidenciar uma possível degradação (CASTELLINI et al., 2013).

QUADRO 1. Indicadores de qualidade físico-hidrica do solo e suas respectivas faixas ótimas ou limites críticos de acordo com Reynolds et al. (2007; 2009)

\begin{tabular}{|c|c|}
\hline $\begin{array}{l}\text { Indicador de qualidade física do } \\
\text { solo }\end{array}$ & Valores de referência \\
\hline Densidade do solo, Ds $\left(\mathrm{Mg} \mathrm{m}^{-3}\right)$ & $\begin{array}{ll}\checkmark & \text { Ótimo: } 0,9 \leq \mathrm{DS} \leq 1,2 \\
\checkmark & \text { Próximo ao ideal: } 0,85 \leq \mathrm{DS}<0,9 \text { e } \\
& 1,2<\mathrm{DS} \leq 1,25 \\
\checkmark & \text { Limites críticos: } 0,85<\mathrm{DS} \text { e DS }>1,25\end{array}$ \\
\hline $\begin{array}{l}\text { Teor de carbono orgânico, } \mathrm{CO}\left(\mathrm{g} \mathrm{kg}^{-}\right. \\
\text {1) }\end{array}$ & $\begin{array}{l}\checkmark \text { Ótimo: } 30 \leq \mathrm{CO} \leq 50 \\
\checkmark \quad \text { Intermediário: } 23 \leq \mathrm{CO}<30 \text { e } 50<\mathrm{CO} \\
\leq 60 \\
\checkmark \text { Más condições: } \mathrm{CO}<23 \text { e } \mathrm{CO}>60\end{array}$ \\
\hline $\begin{array}{l}\text { Índice de estabilidade estrutural, IES } \\
(\%)\end{array}$ & $\begin{array}{ll}\checkmark & \text { Estrutura estável: IES >9 } \\
\checkmark & \text { Baixo risco de degradação } \\
& \text { estrutural: }\end{array}$ \\
\hline
\end{tabular}

AGRARIAN ACADEMY, Centro Científico Conhecer - Goiânia, v.5, n.9; p.261 


\begin{tabular}{|c|c|}
\hline$I E S=\frac{1,724 \times C O(\%)}{\text { Silte }(\%)+\operatorname{Argila}(\%)} \times 100$ & 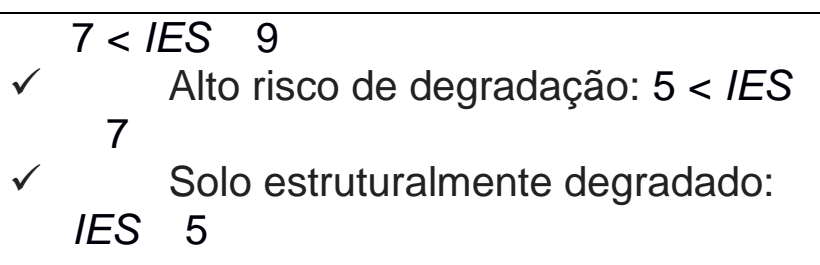 \\
\hline $\begin{array}{l}\text { Condutividade hidráulica saturada, } \\
\mathrm{Ks}\left(\mathrm{mm} \mathrm{s}^{-1}\right)\end{array}$ & $\begin{array}{l}\checkmark \text { Ótimo: } 0,005 \leq K s \leq 0,05 \\
\checkmark \text { Próximo ao ideal: } 0.001<K s \leq 0,005 \text { e } \\
0,05<K s \leq 0,1 \\
\checkmark \text { Pobre: } K s<0,001 \text { e } K s>0,1\end{array}$ \\
\hline $\begin{array}{l}\text { Macroporosidade, } \operatorname{Pmac}\left(\mathrm{m}^{3} \mathrm{~m}^{-3}\right) \\
\mathrm{P}_{\mathrm{mac}}=\theta_{\mathrm{s}}-\theta_{\mathrm{m}}\end{array}$ & $\begin{array}{ll}\checkmark & \text { Ótimo: } P m a c \geq 0,07 \\
\checkmark & \text { Intermediario: 0,04 } \leq \text { Pmac }<0,07 \\
\checkmark & \text { Pobre: } P \text { mac }<0,04\end{array}$ \\
\hline $\begin{array}{l}\text { Capacidade de aeração, } C A\left(\mathrm{~m}^{3} \mathrm{~m}^{-3}\right) \\
\mathrm{CA}=\theta_{\mathrm{S}}-\theta_{\mathrm{FC}}\end{array}$ & $\begin{array}{ll}\checkmark & \text { Ótimo: } C A>0,14 \\
\checkmark & \text { Intermediario: } 0,10 \leq C A \leq 0,14 \\
\checkmark & \text { Pobre: } C A<0,10\end{array}$ \\
\hline $\begin{array}{l}\text { Capacidade de áqua disponível para } \\
\text { planta, } C A D P\left(\mathrm{~m}^{3} \mathrm{~m}^{-3}\right) \\
\text { CADP }=\theta_{\mathrm{FC}}-\theta_{\mathrm{PWP}}\end{array}$ & $\begin{array}{ll}\checkmark & \text { Ideal: } C A D P \geq 0,20 \\
\checkmark & \text { Boa: } 0,15 \leq C A D P<0,20 \\
\checkmark & \text { Limite: } 0,10 \leq C A D P<0,15 \\
\checkmark & \text { Pobre: } C A D P<0,10\end{array}$ \\
\hline $\begin{array}{l}\text { Capacidade de campo relativa, CCR } \\
\text { (adimensional) } \\
C C R=\frac{\theta_{F C}}{\theta_{s}}\end{array}$ & $\begin{array}{l}\checkmark \text { Ótimo: } 0,6 \leq C C R \leq 0,7 \\
\checkmark \text { Solo limitado em água: } C C R<0,6 \\
\checkmark \text { Aeração do solo limitada: } C C R>0,7\end{array}$ \\
\hline
\end{tabular}

$\theta_{\mathrm{S}}=$ teor de água saturada no solo; $\theta_{\mathrm{m}}=$ teor de água matricial do solo $(h=-10 \mathrm{~cm})$; $\theta_{\mathrm{FC}}=$ teor de água no solo na capacidade de campo $(h=-100 \mathrm{~cm}) ; \theta_{\mathrm{PWP}}=$ teor de água no solo no ponto de murcha permanente $(h=-15300 \mathrm{~cm})$.

Diante dessa variedade de indicadores de sustentabilidade associados à qualidade do solo, há também índices estabelecidos com base no crescimento e produção das espécies forrageiras nas áreas de pastagens. Visto que a maior eficiência no acúmulo de fitomassa associada à conservação dos recursos naturais assegura o desenvolvimento sustentável, e também garante uma maior segurança econômica da atividade pecuária, tornam-se indispensáveis avaliações que relacionem o conjunto de indicadores com base na qualidade do solo e na produção de forragem, pois somente através das relações entre eles é possível estabelecer um padrão de respostas dos sistemas e então determinar a sustentabilidade da produção. 
Nesse sentido, Dias-Filho (2014) ressaltou que a caracterização de indicadores de degradação de uma pastagem deve ser relativa à produtividade das espécies forrageiras. As características visuais como, por exemplo, a presença de plantas daninhas e de solo descoberto são indicadores secundários de degradação das áreas de pastagem (DIAS-FILHO, 2011). Deste modo, conforme Dias-Filho (2011), a capacidade de suporte seria um indicador importante da degradação e, consequentemente, da insustentabilidade produtiva de uma determinada área, uma vez que a taxa de lotação em uma pressão de pastejo ótima é determinada pela oferta de forragem.

\section{CONSIDERAÇÕES FINAIS}

O fenômeno de degradação das pastagens é considerado um dos maiores entraves para a atividade pecuária. Por esta razão, é extremamente importante determinar a sustentabilidade dos diferentes sistemas de produção, por meio de indicadores sensíveis as alterações do manejo, pois somente através da utilização desses índices é possível caracterizar esses sistemas e revelar suas vantagens e desvantagens através do tempo, de modo a subsidiar um manejo que permita a recuperação dessas áreas.

Desse modo, estudos sobre a utilização de indicadores de sustentabilidade estabelecidos com base na qualidade do solo e acúmulo de fitomassa, tornam-se fundamentais para dar subsidio ao planejamento dos sistemas produtivos em diferentes regiões e garantir a contínua sustentabilidade e conservação desses ecossistemas.

\section{REFERÊNCIAS}

ABDALLA, M.; HASTINGS, A.; CHADWICK, D. R.; JONES, D. L.; EVANS, C.D. et al. Critical review of the impacts of grazing intensity on soil organic carbon storage and other soil quality indicators in extensively managed grasslands. Agriculture, Ecosystems \& Environment, v. 253, p. 62-81, 2018. Disponível em: <http://dx.doi.org/10.1016/j.agee.2017.10.023> DOI: 10.1016/j.agee.2017.10.023

ALVES, M. C.; SUZUKI, L. G. A. S.; SUZUKI, L. E. A. S. Densidade do solo e infiltração de água como indicadores da qualidade física de um latossolo vermelho distrofíco em recuperação. Revista Brasileira de Ciência do Solo. 31:617-625, 2007. Disponível em: <http://dx.doi.org/10.1590/S0100-06832007000400002> DOI: $10.1590 /$ S0100-06832007000400002

ALEXANDRIDIS, T. K., SOTIROPOULOU, A. M., BILAS, G., KARAPETSAS, N.; SILLEOS, N. G. The Effects of Seasonality in Estimating the C-Factor of Soil Erosion Studies. Land Degradation \& Development, v. 26, n. 6, p. 596-603, 2013. Disponível em: <https://doi.org/10.1002/ldr.2223> DOI: 10.1002/ldr.2223

ATUCHA, A.; MERWIN, I. A.; BROWN, M. G.; GARDIAZABAL, F.; MENA, F.; ADRIAZOLA, C.; LEHMANN, J. Soil erosion, runoff and nutrient losses in an avocado (Persea americana Mill) hillside orchard under different groundcover management systems. Plant and Soil, v. 368, n. 1-2, p. 393-406, 2013. Disponível em: < https://link.springer.com/article/10.1007/s11104-012-1520-0> DOI: 10.1007/s11104012-1520-0 
ANGASSA, A.; OBA, G. Effects of grazing pressure, age of enclosures and seasonality on bush cover dynamics and vegetation composition in southern Ethiopia. Journal of Arid Environments, v. 74, n. 1, p. 111-120, 2010. Disponível em: $\quad$ <https://doi.org/10.1016/j.jaridenv.2009.07.015> 10.1016/j.jaridenv.2009.07.015

AGUIAR, M. I.; MAIA, S. M. F.; SILVA XAVIER, F. A.; SÁ MENDONÇA, E.; ARAÚJO FILHO, J. A.; DE OLIVEIRA, T. S. Sediment, nutrient and water losses by water erosion under agroforestry systems in the semi-arid region in northeastern Brazil. Agroforestry systems, v. 79, n. 3, p. 277-289, 2010. Disponível em: <https://link.springer.com/article/10.1007/s10457-010-9310-2> DOI: 10.1007/s10457010-9310-2

AGNESE, C., BAGARELlO, V., BAIAMONTE, G., IOVINO, M., 2011. Comparing physical quality of forest and pasture soils in a Sicilian watershed. Soil Science Society of America J. 75, 1958-1970. Disponível em: DOI: 10.2136/sssaj2011.0044

BALBINO, L.C; BARCELLOS, A.O; STONE, L. F. Marco referencial: integração lavoura-pecuária-floresta. Embrapa Cerrados-Livro científico (ALICE), 2011. Disponível

em: <https://www.alice.cnptia.embrapa.br/bitstream/doc/923530/1/balbino01.pdf>

BAGARELLO, V.; DI PRIMA, S.; IOVINO, M.; PROVENZANO, G.; SGROI, A. Testing diferente approaches to characterize Burundian soils by the BEST procedure. Geoderma 162, 141-150, 2011. Disponível em: <https://doi.org/10.1016/j.geoderma.2011.01.014> 10.1016/j.geoderma.2011.01.014

BERTOL, I.; BEUTLER, J.F.; LEITE, D.; BATISTELA, O. Propriedades físicas de um Cambissolo Húmico afetadas pelo tipo de manejo do solo. Scientia Agricola, v.58, n. 3, p.555-560, 2001. Disponível em: <http://dx.doi.org/10.1590/S010390162001000300018> DOI: 10.1590/S0103-90162001000300018

BERTOL, I., ZOLDAN JUNIOR, W. A.; GONZÁlEZ, A. P.; BARBOSA, F. T.; WERNER, R. S. Sediment transport in runoff on rugous soil surface submitted to simulated rainfall. Scientia Agricola, v. 67, n. 5, p. 591-597, 2010. Disponível em: <http://dx.doi.org/10.1590/S0103-90162010000500013> DOI: 10.1590/S010390162010000500013

BORLACHENCO, N. G. C.; GONÇALVES, A. B. Expansão agrícola: elaboração de indicadores de sustentabilidade nas cadeias produtivas de Mato Grosso do Sul. Interações (Campo Grande), v. 18, n. 1, p. 119-128, 2017. Disponível em: <http://dx.doi.org/10.20435/1984-042X-2017-v.18-n.1(09)> DOI: 10.20435/1984042X-2017

BUDAK, M., GUNAL, H., CELIK, I., YILDIZ, H., ACIR, N., \& ACAR, M. Soil quality assesment of upper tigris basin. Carpathian journal of earth and environmental sciences, v. 13, n. 1, p. 301-316, 2018. Disponível em: < 
CÂNDIDO, G. A.; NÓBREGA, M. M.; FIGUEIREDO, M. T. M.; SOUTO MAIOR, M. M. Avaliação da sustentabilidade de unidades de produção agroecológicas: um estudo comparativo dos métodos IDEAS e MESMIS. Ambiente \& Sociedade, v. 18, n. 3, p. 99-120, 2015. Disponível em: <http://dx.doi.org/10.1590/18094422ASOC756V1832015> DOI: 10.1590/1809-4422ASOC756V1832015

CARDOSO, E. L.; SILVA, M.L.N.; CURI, N.; FERREIRA, M.M.; FREITAS, D.A.F. Qualidade química e física do solo sob vegetação arbórea nativa e pastagens no Pantanal Sul-Mato Grossense. Revista Brasileira de Ciência do Solo, v. 35, n. 2, p. 613-622, $2011 . \quad$ Disponível em: <http://www.scielo.br/pdf/rbcs/v35n2/v35n2a30.pdf>

CASTELLINI, M.; PIRASTRU, M.; NIEDDA, M.; VENTRELLA, D. Comparing physical quality of tilled and no-tilled soils in an almond orchard in South Italy. Italian Journal of Agronomy, 8, 149-157, 2013. Disponível em: <https://doi.org/10.4081/ija.2013.e20> DOI: 10.4081/ija.2013.e20

CASTELLINI, M et al. Use of BEST procedure to assess soil physical quality in the Baratz lake catchment (Sardinia, Italy). Soil Science Society of America Journal, v. 80 , n. 3, p. $742-755,2016$. Disponível em: <http://eprints.uniss.it/11462/> DOI: $10.2136 /$ sssaj2015.11.0389

CANELLAS, L.P.; VELLOSO, A.C.X.; MARCIANO, C.R.; RAMALHO, J.F.G.P.; RUMJANEK, V.M.; REZENDE, C.E.; SANTOS, G.A. Propriedades químicas de um Cambissolo cultivado com cana-de-açúcar, com preservação do palhiço e adição de vinhaça por longo tempo. Revista Brasileira de Ciência do Solo, v. 27, n. 5, p.935944, 2003. Disponível em: <http://dx.doi.org/10.1590/S010006832003000500018 > DOI: 10.1590/S0100-06832003000500018

CARVALHO, W. T. V., MINIGHIN, D. C., GONÇALVES, L. C., VILLANOVA, D. F. Q., MAURICIO, R. M., \& PEREIRA, R. V. G. Pastagens degradadas e técnicas de recuperação: Revisão. PUBVET, v. 11, p. 0947-1073, 2017. Disponível em: <http://dx.doi.org/10.22256/PUBVET.V11N10.1036-1045> 10.22256/PUBVET.V11N10.1036-1045

CERDÀ, A.; LAVEE, H.; ROMERO-DÍAZ, A.; HOOKE, J.; MONTANARELLA, L. 2010. Soil erosion and degradation in Mediterranean-type ecosystems. Preface. Land Degradation \& Development 21(2), 71-74. Disponível em: $<$ http://dx.doi.org/10.1002/ldr.968> DOI: 10.1002/ldr.968

DAVIDSON, E.A.; SAVAGE, K.; VERCHOT, L.V.; NAVARRO, R. Mimimizing artifacts and biases in chamber-based measurements of soil respiration. Agricultural and Forest Meteorology, v.113, p.21-37, 2002. Disponível em: <https://doi.org/10.1016/S0168-1923(02)00100-4> DOI: 10.1016/S01681923(02)00100-4 
DERECZYNSKI, C.; SILVA, W.L.; MARENGO, J. Detection and projections of climate change in Rio de Janeiro, Brazil. American Journal of Climate Change, v. 2, n. 1, p. 25-33, 2013. Disponível em: < http://file.scirp.org/pdf/AJCC_2013032715394029.pdf> DOI: 10.4236/ajcc.2013.21003

DEFERSHA, M. B.; QURAISHI, S.; MELESSE, A. Interrill erosion, runoff and sediment size distribution as affected by slope steepness and antecedent moisture content. Hydrology and Earth System Sciences Discussions, v. 7, n. 4, p. 64476489, 2010. Disponível em: <http://www.hydrol-earth-syst-sci.net/15/2367/2011/> DOI: $10.5194 /$ hess-15-2367-2011

DIAS-FILHO, M. B. Degradação de pastagens: processos, causas e estratégias de recuperação. 3. ed. Belém: Embrapa Amazônia Oriental. 190 p. 2007. Disponível em: < http://www.diasfilho.com.br/Livro/Degradacao_pastagens-Moacyr_DiasFilho.pdf>

DIAS-FILHO, M. B. Degradação de pastagens: processos, causas e estratégias de recuperação. 4. ed. rev., atual. e ampl. Belém, PA, 2011. Disponível em: < https://www.researchgate.net/publication/261026141_Degradacao_de_pastagens_pr ocessos_causas_e_estrategias_de_recuperacao?enrichld=rgreqf23596569f187e0639bcf8c12b4ebe34-

XXX\&enrichSource=Y292ZXJQYWdIOzI2MTAyNjE0MTtBUzoxMDE2NDcxMzI5MjE4 ODFAMTQwMTIONTk3Mzk4NA\%3D\%3D\&el=1_x_2\&_esc=publicationCoverPdf>

DIAS-FILHO, M. B. Diagnóstico das pastagens no Brasil. Embrapa Amazônia Oriental-Documentos (INFOTECA-E), Belém, PA, 2014, p.38. Disponível em: < https://www.infoteca.cnptia.embrapa.br/bitstream/doc/986147/1/DOC402.pdf>

FREDDI, O.S.; CENTURION, J.F.; BEUTLER, A.N.; ARATANI, R.G.; LEONEL, C.L. Compactação do solo no crescimento radicular e produtividade da cultura do milho. Revista Brasileira de Ciência do Solo, v. 31, n. 4, p. 627-636, 2007. Disponível em: < http://dx.doi.org/10.1590/S0100-06832007000400003> DOI: 10.1590/S010006832007000400003

FORTIN, G.; LEBLANC, M.; SCHIAVONE, S.; CHOUINARD, O.; UTZSCHNEIDER, A. Local perceptions, Ruslefac mapping, and field results: The sediment budget of Cocagne River, New Brunswick, Canada. Environmental management, v. 55, n. 1, p. 113-127, 2015. Disponível em: < https://www.semanticscholar.org/paper/Localperceptions\%2C-RUSLEFAC-mapping\%2C-and-field-the-Fortin-

Leblanc/858334d283f16e6bf3242ae654f3bd129e3e30fc> DOI: 10.1007/s00267-0140399-3

FREITAS, L.; OLIVEIRA, I. A.; SILVA, L. S.; FRARE, C. V.; FILLA, V. A. et al. Indicadores da qualidade química e física do solo sob diferentes sistemas de manejo. Revista Unimar Ciências, v. 26, n. 1-2, 2017. Disponível em: <http://ojs.unimar.br/index.php/ciencias/article/view/511/278>

GALDINO, S.; SANO, E. E.; ANDRADE, R. G.; GREGO, C. R.; NOGUEIRA, S. F. et al. Large-scale Modeling of Soil Erosion with RUSLE for Conservationist Planning of 
Degraded Cultivated Brazilian Pastures. Land Degradation \& Development, v. 27, n. 3, p. 773-784, 2016. Disponível em: <https://doi.org/10.1002/ldr.2414> DOI: $10.1002 / \mathrm{ldr} .2414$

GARCEZ, G. S. O princípio da solidariedade intergeracional como pressuposto para a adoção de um paradigma ambiental de sustentabilidade. Interfaces: Revista de Saúde, Meio Ambiente e Sustentabilidade, v. 9, n. 1, p. 109-114, 2014. Disponível em: $\quad<$ http://www3.sp.senac.br/hotsites/blogs/InterfacEHS/o-principio-dasolidariedade-intergeracional-como-pressuposto-para-a-adocao-de-um-paradigmaambiental-de-sustentabilidade/>

GONZALEZ-SOSA, E.; BRAUD, I.; DEHOTIN, J.; LASSABATÈRE, L.; ANGULOJARAMILLO, R. et al. Impact of land use on the hydraulic properties of the topsoil in a small French catchment. Hydrological Processes, v. 24, n. 7, p. 2382-2399, 2010. Disponível em: <https://doi.org/10.1002/hyp.7640> DOI: 10.1002/hyp.7640

GUIMARÃES, N. F.; GALlO, A. S.; SANTOS, C. C.; MORINIGO, K. P. G.; BENTOS, A. B.; CARVALHO, E. M. Avaliação da sustentabilidade de um agroecossistema pelo método MESMIS. Scientia Plena, v. 11, n. 5, p. 1-11, 2015. Disponível em: <https://www.scientiaplena.org.br/sp/article/view/1993/1212>

HAN, J. G.; ZHANG, Y. J.; WANG, C. J.; BAI, W. M.; WANG, Y. R. et al. Rangeland degradation and restoration management in China. The Rangeland Journal, v. 30, n. 2, p. 233-239, 2008. Disponível em: <https://doi.org/10.1071/RJ08009> DOI: 10.1071/RJ08009

HOU, F. J.; NAN, Z. B.; XIE, Y. Z.; LI, X. L.; LIN, H. L. et al. Integrated crop-livestock production systems in China. The Rangeland Journal, v. 30, n. 2, p. 221-231, 2008. Disponível em: <https://doi.org/10.1071/RJ08018> DOI: 10.1071/RJ08018

IOVINO, M., CASTELLINI, M., BAGARELLO, V., GIORDANO, G. Using static and dynamic indicators to evaluate soil physical quality in a Sicilian area. Land Degradation and Development, v. 72, p. 200-210, 2016. Disponível em: <https://doi.org/10.1002/ldr.2263> DOI: 10.1002/ldr.2263

JARVIE, H. P.; SHARPLEY, A. N.; WITHERS, P. J.; SCOTT, J. T.; HAGGARD, B. E.; NEAL, C. Phosphorus mitigation to control river eutrophication: Murky waters, inconvenient truths, and "postnormal" science. Journal of Environmental Quality, v. 42, n. 2, p. 295-304, 2013. Disponível em: < https://www.ncbi.nlm.nih.gov/pubmed/23673821 > DOI: 10.2134/jeq2012.0085

JEMAI, I.; AISSA, N. B.; GUIRAT, S. B.; BEN-HAMMOUDA, M.; GALLALI, T. Impact of three and seven years of no-tillage on the soil water storage, in the plant root zone, under a dry subhumid Tunisian climate. Soil and Tillage Research, v. 126, p. 26-33, 2013. Disponível em: <https://doi.org/10.1016/j.still.2012.07.008> DOI: 10.1016/j.still.2012.07.008

JING, Z.; CHENG, J.; SU, J.; BAI, Y.; JIN, J. Changes in plant community composition and soil properties under 3-decade grazing exclusion in semiarid 
grassland. Ecological Engineering, v. 64, p. 171-178, 2014. Disponível em: <https://doi.org/10.1016/j.ecoleng.2013.12.023> DOI: 10.1016/j.ecoleng.2013.12.023

JIMENEZ, R.L.; GONÇALVES, W.G.; ARAÚJO FILHO, J.V.; ASSIS, R.L.; PIRES, F.R.; SILVA, G.P. Crescimento de plantas de cobertura sob diferentes níveis de compactação em um Latossolo Vermelho. Revista Brasileira de Engenharia Agrícola e Ambiental, v. 12, n. 2, p. 116-121, 2008. Disponível em: <http://dx.doi.org/10.1590/S1415-43662008000200002> DOI: 10.1590/S141543662008000200002

KELISHADI H.; MOSADDEGHI M.R.; HAJABBASI M.A.; AYOUBI S. Near-saturated soil hydraulic properties as influenced by land use management systems in Koohrang region of central Zagros, Iran. Geoderma 213, pp. 426-434, 2014. Disponível em: <https://doi.org/10.1016/j.geoderma.2013.08.008> DOI: 10.1016/j.geoderma.2013.08.008

KOTLYAROVA, E. G.; CHERNIAVSKIH, V. I.; DUMACHEVA, E. V. Ecologically Safe Architecture of Agrolandscape is basis for sustainable development. Sustainable Agriculture Research, v. 2, n. 2, p. 11, 2013. Disponível em: <http://dx.doi.org/10.5539/sar.v2n2p11 > DOI: 10.5539/sar.v2n2p11

KUZMA, E.L.; DOLIVEIRA, S. L. D.; ATAMANCZUK, M. J.; CARDOSO, A. A. O Perfil Financeiro das Empresas Aderentes e Não Aderentes ao Índice de Sustentabilidade Empresarial da BM\&FBOVESPA. Organizações e Sustentabilidade, v. 3, n. 1, p. 47-78, 2015. Disponível em: <http://dx.doi.org/10.5433/2318-9223.2015v3n1p47> DOI: $10.5433 / 2318-9223.2015 v 3 n 1 p 47$

LAURINDO, M. C. O.; NÓBREGAS, L. H. P.; PEREIRA, J. O.; MELO, D. \& LAURINDO, E. L. Atributos físicos do solo e teor de carbono orgânico em sistemas de plantio direto e cultivo mínimo. Engenharia na Agricultura, viçosa - MG, V.17 N.5, $\quad$ p. $367 \quad-\quad 374, \quad 2009 . \quad$ Disponível em: <https://reveng.ufv.br/index.php/reveng/article/view/163/78>

LALIBERTÉ, E.; SHIPLEY, B.; NORTON, D. A.; SCOTT, D. Which plant traits determine abundance under long-term shifts in soil resource availability and grazing intensity?. Journal of Ecology, v. 100, n. 3, p. 662-677, 2012. Disponível em: <https://doi.org/10.1111/j.1365-2745.2011.01947.x> DOI: $10.1111 / \mathrm{j} .1365-$ 2745.2011.01947.x

LIMA, C. L. R.; de PILLON, C. N.; LIMA, A. C. R de. Qualidade Física do Solo: Indicadores quantitativos. Documentos, 196. Pelotas: Embrapa Clima Temperado, 25p., 2007. Disponível em: <https://www.embrapa.br/clima-temperado/busca-depublicacoes/-/publicacao/745869/qualidade-fisica-do-solo-indicadores-quantitativos>

MASTO, R. E.; CHHONKAR, P. K.; SINGH, D.; PATRA, A. K. Alternative soil quality indices for evaluating the effect of intensive cropping, fertilisation and manuring for 31 years in the semiarid soils of India. Environmental monitoring and assessment, v. 136, n. 1-3, p. 419-435, 2008 . Disponível em: <https://link.springer.com/article/10.1007\%2Fs10661-007-9697-z> DOI: 10.1007/s10661-007-9697-z 
MARZAIOLI, R.; ASCOLI, R. D.; DE PASCALE, R. A.; RUTIGLIANO, F. A. Soil quality in a Mediterranean area of Southern Italy as related to different land use types. Applied Soil Ecology, v. 44, p. 205- 212, 2010. Disponível em: <https://doi.org/10.1016/j.apsoil.2009.12.007> DOI: 10.1016/j.apsoil.2009.12.007

MOREIRA, F. R.; DECHEN, S. C. F.; SILVA, Á. P. D.; FIGUEIREDO, G. C.; MARIA, I. C. D.; PESSONI, P. T. Least limiting water range in an Oxisol cultivated under a no-tillage system for 25 years. Revista Brasileira de Ciência do Solo, v. 38, n. 1, p. 118-127, 2014. Disponível em: <http://dx.doi.org/10.1590/S010006832014000100011 > DOI: 10.1590/S0100-06832014000100011

MUKHERJEE, A; LAL, R. Comparison of soil quality index using three methods. PLoS One, v. 9, n. 8, p. e105981, 2014. Disponível em: $<$ https://doi.org/10.1371/journal.pone.0105981 > DOI: 10.1371/journal.pone.0105981

ODUM, E. P. \& BARRETT, G. W. Fundamentos da Ecologia. Tradução Pégasus Sistemas e Soluções. São Paulo: Cengage Learning, 612p., 2011. Disponível em: $<$

https://www.bdpa.cnptia.embrapa.br/consulta/busca?b=ad\&id=898706\&biblioteca=C NPAB\&busca=autoria:\%22ODUM,\%20E.P.\%22\&qFacets=autoria:\%22ODUM,\%20E. P.\%22\&sort=\&paginacao=t\&paginaAtual $=1>$

ODRIOZOLA, I.; GARCÍA-BAQUERO, G.; FORTIN, M. J.; LASKURAIN, N. A.; ALDEZABAL, A. Grazing exclusion unleashes competitive plant responses in Iberian Atlantic mountain grasslands. Applied Vegetation Science, v. 20, n. 1, p. 50-61, 2017. Disponível em: <https://doi.org/10.1111/avsc.12277> DOI: 10.1111/avsc. 12277

OLIVEIRA, A. H., SILVA, M. L. N., CURI, N., AVANZI, J. C., KLINKE NETO, G., \& ARAÚJO, E. F. Water erosion in soils under eucalyptus forest as affected by development stages and management systems. Ciência e Agrotecnologia, v. 37, n. 2, p. 159-169, 2013. Disponível em: <http://dx.doi.org/10.1590/S141370542013000200007> DOI: 10.1590/S1413-70542013000200007

OLIVEIRA, J. T. C.; FIGUEREDO, E. F.; DINIZ, W. P. D. S.; OLIVEIRA, L. F. P. D.; ANDRADE, P. A. M. D. et al. Diazotrophic Bacterial Community of Degraded Pastures. Applied and Environmental Soil Science, v. 2017, 2017. Disponível em: <https://doi.org/10.1155/2017/2561428> DOI: 10.1155/2017/2561428

OZALP, M.; YUKSEL, E.E.; YUKSEK, T. Soil property changes after the conversion from forest to pasture in the mount Sacinka, Artvin, Turkey. Land Degradation and Development, 2015. Disponível em: <https://doi.org/10.1002/ldr.2353> DOI: 10.1002/ldr.2353

PAUSTIAN, K.; SIX, J.; ELLIOT, E.T.; HUNT, H.W. Management options for reducing $\mathrm{CO}_{2}$ emissions from agricultural soils. Biogechemistry, v. 48, p. 147-163, 2000. <https://link.springer.com/article/10.1023/A:1006271331703> DOI: 10.1023/A:1006271331703 
PARKIN, T.B.; DORAN, J.W.; FRANCO-VIZCAÍNO, E. Field and Laboratory Tests of Soil Respiration. In: DORAN, J.W. et al. Methods for assessing soil quality. Madison: Soil Science Society of America, 1996. p.231-245. Disponível em: <http://agris.fao.org/agris-search/search.do?recordID=US201300917254>

PASSOS, R. R.; DA COSTA, L. M.; DE ASSIS, I. R.; SANTOS, D. A.; RUIZ, H. A.; GUIMARÃES, L. A. D. O. P.; ANDRADE, F. V. Least limiting water range of Udox soil under degraded pastures on different sun-exposed faces. International Agrophysics, v. 31, n. 3, p. 393-400, 2017. Disponível em: <https://doi.org/10.1515/intag-2016-0066> DOI: 10.1515/intag-2016-0066

PIERI, C.J.M.G. Fertility of soils: A future for farming in the West African savannah. Springer-Verlag, Berlin, 1992. Disponível em: <https://www.springer.com/us/book/9783642843228>

PIMENTEL, D.; HARVEY, C.; RESOSUDARMO, P.; SINCLAIR, K.; KURZ, D. et al. Environmental and economic costs of soil erosion and conservation benefits. Science, v. 267, n. 5201, p. 1117-1123, 1995. Disponível em: <https://www.ncbi.nlm.nih.gov/pubmed/17789193> 10.1126/science.267.5201.1117

PINHEIRO MACHADO, L. C. Pastoreio racional voisin: tecnología agroecológica para o $3^{\circ}$ milenio. $2^{\circ}$ edição, Editora Expressão Popular, São Paulo, 376 p., 2010.Disponível em:

https://books.google.com.br/books/about/Pastoreio_racional_voisin.html?id=KCX0Gg AACAAJ\&redir_esc $=\mathrm{y}>$

RALISCH, R.; ALMEIDA, E.; SILVA, A. P.; PEREIRA NETO, O. C.; GUIMARÃES, M. F. Morphostructural characterization of soil conventionally tilled with mechanized and animal traction with and without cover crop. Revista Brasileira de Ciência do Solo, v. 34, p. 1795-1802, 2010. Disponível em: <http://dx.doi.org/10.1590/S010006832010000600003> DOI: 10.1590/S0100-06832010000600003

RANGEL, O.J.P.; SILVA, C.A. Estoques de carbono e nitrogênio e frações orgânicas de Latossolo submetido a diferentes sistemas de uso e manejo. Revista Brasileira de Ciência do Solo, v. 31, n. 80, p. 1609-1623, 2007. Disponível em: < http://dx.doi.org/10.1590/S0100-06832007000600037> DOI: 10.1590/S010006832007000600037

REGO, C. A. R. M et al. Cost Analysis of Corn Cultivation in the Setup of the CropLivestock-Forest Integration System to Recover Degraded Pastures. Journal of Agricultural Science, v. 9, n. 6, p. 168, 2017. Disponível em: < https://www.embrapa.br/busca-de-publicacoes/-/publicacao/1074283/estoques-decarbono-e-nitrogenio-no-solo-em-funcao-da-cronossequencia-da-pastagem-aposser-formada-em-sistema-ilpf>

REN, W.; HU, N.; HOU, X.; ZHANG, J.; GUO, H. et al. Long-term overgrazinginduced memory decreases photosynthesis of clonal offspring in a perennial grassland plant. Frontiers in Plant Science, v. 8, p. 419, 2017. Disponível em: 
<https://www.ncbi.nlm.nih.gov/pmc/articles/PMC5401901/>

DOI: 10.3389/fpls.2017.00419

REICHERT, J.M; KAISER, D.R.; REINERT, D.J.; RIQUELME, U.F.B. Variação temporal de propriedades físicas do solo e crescimento radicular de feijoeiro em quatro sistemas de manejo. Pesquisa Agropecuária Brasileira, v. 44, n. 3, p. 310319, 2009. Disponível em: < http://dx.doi.org/10.1590/S0100-204X2009000300013> DOI: 10.1590/S0100-204X2009000300013

REYNOLDS, W.D.; DRURY, C.F.; YANG, X.M.; FOX, C.A.; TAN, C.S.; ZHANG, T.Q. Land management effects on the near-surface physical quality of a clay loam soil. Soil and Tillage Research, v. 96, p. 316-330, 2007. Disponível em: <https://doi.org/10.1016/j.still.2007.07.003> DOI: 10.1016/j.still.2007.07.003

REYNOLDS, W.D.; DRURY, C.F.; TAN, C.S.; FOX, C.A.; YANG, X.M. Use of indicators and pore volume-function characteristics to quantify soil physical quality. Geoderma, v. 152, n. 3-4, p. 252-263, 2009. Disponível em: $<$ https://doi.org/10.1016/j.geoderma.2009.06.009> 10.1016/j.geoderma.2009.06.009

REYNOLDS, W.D.; DRURY, C.F.; YANG, X.M.; TAN, C.S. Optimal soil physical quality inferred through structural regression and parameter interactions. Geoderma, v. $146, \quad$ n. $3-4, \quad$ p. $466-474,2008$. Disponível em: <https://doi.org/10.1016/j.geoderma.2008.06.017> 10.1016/j.geoderma.2008.06.017

REYNOLDS, W.D.; BOWMAN, B.T.; DRURY, C.F.; TAN, C.S.; LU, X. Indicators of good soil physical quality: density and storage parameters. Geoderma, v. 110, n. 12, p. 131-146, 2002. Disponível em: <https://doi.org/10.1016/S0016-7061(02)002288> DOI: 10.1016/S0016-7061(02)00228-8

ROCHA JUNIOR, P. R.; ANDRADE, F. V.; DE SÁ MENDONÇA, E.; DONAGEMMA, G. K.; FERNANDES, R. B. A.; BHATTHARAI, R.; \& KALITA, P. K. Soil, water, and nutrient losses from management alternatives for degraded pasture in Brazilian Atlantic Rainforest biome. Science of the Total Environment, v. 583, p. 53-63, 2017. Disponível em: <https://doi.org/10.1016/j.scitotenv.2016.12.187> DOI: 10.1016/j.scitotenv.2016.12.187

SALAZAR A; BALDI G; HIROTA M; SYKTUS J; MCALPINE C. Land use and land cover change impacts on the regional climate of non-Amazonian South America: a review. Global Planet Change, 128:103-119, 2015. Disponível em: <https://doi.org/10.1016/j.gloplacha.2015.02.009> 10.1016/j.gloplacha.2015.02.009

SATTLER, D; SELIGER, R; NEHREN, U; TORRES, F. N; SILVA, A. S; RAEDIG, C; HEINRICH, J. Pasture Degradation in South East Brazil: Status, Drivers and Options for Sustainable Land Use Under Climate Change. In: Climate Change Adaptation in Latin America. Springer, Cham, 2018. p. 3-17. Disponível em: < https://link.springer.com/chapter/10.1007/978-3-319-56946-8_1> 
SANT'ANNA A.C et al. Bem-estar animal: um dos critérios da sustentabilidade na pecuária. In: BARBOSA B.C et al. (Orgs.) Tópicos em Sustentabilidade \& Conservação. 1. ed. Juiz de Fora, MG: Edição dos autores, p. 17-46, 2017. Disponível em: < http://www.fazendatriqueda.com.br/wpcontent/uploads/2018/05/BARBOSA-et-al-2017_T\%C3\%B3picos-em-

Sustentabilidade-Conserva\%C3\%A7\%C3\%A3o_Book-1.pdf>

SILVA, D. V., MACIEL, K. N., SANTOS, J. R., MENDES, J. G., \& BARBOSA, L. C. B. G. Agroecologia e Convivência com o Semiárido Brasileiro: uma análise preliminar. Diversitas Journal, v. 3, n. 1, p. 76-84, 2018. Disponível em: < http://www.kentron.ifal.edu.br/index.php/diversitas_journal/article/view/547> DOI: $\underline{10.17648 / \text { diversitas-journal-v3i1.547 }}$

SILVA A.S; BOTELHO R.G.M. Degradação dos solos no estado do Rio de Janeiro. In: Guerra AJT, Jorge MCO (eds) Degradação dos Solos no Brasil. 1ed.Rio de Janeiro: Bertrand Brasil, 2014, v., pp 261-292, 2014. Disponível em: < https://www.researchgate.net/publication/267777300_Degradacao_dos_Solos_no_Br asil>

SILVA, R.R.; SILVA, M.L.N.; FERREIRA, M.M. Atributos físicos indicadores da qualidade do solo sob sistemas de manejo na Bacia Alto do Rio Grande-MG. Ciência e Agrotecnologia, v. 29, n. 4, p. 719-730, 2005. Disponível em: < http://www.scielo.br/pdf/cagro/v29n4/a03v29n4>

SILVA, R. B.; SANTOS, A. C.; BATISTA, R. B. Respiração edáfica como indicativo da qualidade do solo em três agrossistemas. Enciclopédia Biosfera, v. 6, n. 11, p. 1-15, 2010. Disponível em: < http://www.conhecer.org.br/enciclop/2010c/respiracao\%20edafica.pdf>

SOLDÁ, C.C. Avaliação da sustentabilidade em pastagens através de método participativo. 2012. 75 f. Dissertação (mestrado profissional) - Universidade Federal de Santa Catarina, Centro de Ciências Agrárias, Programa de Pós-Graduação em Agroecossistemas, Florianópolis, 2012. Disponível em: < https://repositorio.ufsc.br/handle/123456789/100965>

SHAKESBY, R.A.; BENTO, C.P.M.; FERREIRA, C.S.S.; FERREIRA, A.J.D.; STOOF, C.R. et al. Impacts of prescribed fire on soil loss and soil quality: an assessment based on an experimentally-burned catchment in Central Portugal. Catena 128:
178-193,
2013.
Disponível em:

https://www.sciencedirect.com/science/article/pii/S034181621300074X>

DOI: $\underline{10.1016 / \text { J.catena.2013.03.012 }}$

SMIRNOVA, I; SADANOV, A; SABDENOVA, A; NURMUKHANBETOVA, A; Restoration of Degraded Pasture Soils on the Basis of EM Associations. In: Rhizobium Biology and Biotechnology. Springer, Cham, p. 11-24, 2017. Disponível em: < https://link.springer.com/chapter/10.1007/978-3-319-64982-5_2>

SQUIRES, V. R. (Ed.). Rangeland stewardship in Central Asia: balancing improved livelihoods, biodiversity conservation and land protection. Springer 
Science \& Business Media, 2012. Disponível em: < https://www.springer.com/br/book/9789400753662 >

STRASSBURG, B. B., LATAWIEC, A. E., BARIONI, L. G., NOBRE, C. A., DA SILVA, V. P., VALENTIM, J. F., ASSAD, E. D. When enough should be enough: Improving the use of current agricultural lands could meet production demands and spare natural habitats in Brazil. Global Environmental Change, v. 28, p. 84-97, 2014. Disponível em:

https://www.sciencedirect.com/science/article/pii/S0959378014001046>

DOI: 10.1016/j.gloenvcha.2014.06.001

SOUZA, E. S., ANTONINO, A. C., ANGULO-JARAMILLO, R., \& NETTO, A. M. Caracterização hidrodinâmica de solos: Aplicação do método Beerkan1. R. Bras. Eng. Agríc. Ambiental, v. 12, n. 2, p. 128-135, 2008. Disponível em: < https://www.researchgate.net/profile/Eduardo_Souza13/publication/262502546_Hydr odynamic_characterization_of_soils_application_of_the_Beerkan_method/links/53fa6 92c0cf27c365cf034b8/Hydrodynamic-characterization-of-soils-application-of-theBeerkan-method.pdf>

SOUSA, A. B., COSTA, C. T. F., FIRMINO, P. R. A., SOUZA BATISTA, V. Tecnologias sociais de convivência com 0 semiárido na região do cariri cearense. Cadernos de Ciência \& Tecnologia, v. 34, n. 2, p. 197-220, 2018. Disponível em:

https://seer.sct.embrapa.br/index.php/cct/article/viewFile/26327/14129>

SOARES FILHO, C. V., Monteiro, F. A. \& Corsi, M. Recuperação de pastagens degradadas de Brachiaria decumbens. 2. Variação sazonal de parâmetros bioquímico-fisiológicos. Pasturas Tropicales, 14, 7-13, 1992. Disponível em: < http://ciat-library.ciat.cgiar.org/Articulos_Ciat/Vol14_rev2_a\%C3\%B1092_art3.pdf>

TEAGUE, W. R. et al. Soil and herbaceous plant responses to summer patch burns under continuous and rotational grazing. Agriculture, ecosystems \& environment, v. 137, n. 1-2, p. 113-123, 2010. Disponível em: < https://www.sciencedirect.com/science/article/pii/S0167880910000265> DOI: 10.1016/j.agee.2010.01.010

TOPP, G.C.R., W.D.; COOK. F.J.; KIRBY, J.M.; CARTER, M.R. Physical attributes of soil quality. Development in Soil Science. Elsevier, New York, NY, pp. 21-58, 1997. Disponível em: https://www.sciencedirect.com/science/article/pii/S0166248197800293> DOI: 10.1016/S0166-2481(97)80029-3

TORMENA, C. A., ARAÚJO, M. A., FIDALSKI, J., COSTA, J. D. Variação temporal do intervalo hídrico ótimo de um Latossolo Vermelho distroférrico sob sistemas de plantio direto. Revista Brasileira de Ciência do Solo, v. 31, p. 211-219, 2007. Disponível em: < http://www.scielo.br/pdf/rbcs/v31n2/a03v31n2>

THEODORO, V. C. A. et al. Indicadores ecológicos de sustentabilidade de unidades de produção agrícola do assentamento Facão - Cáceres, MT, Brasil. Revista 
Brasileira de Agroecologia. v. 22 6(3). p.21-33, 2011. Disponível em: < http://revistas.aba-agroecologia.org.br/index.php/rbagroecologia/article/view/9945>

VALENTINI, C. M. A.; ABREU, J. G.; FARIA, R. A. P. G. Respiração do solo como bioindicador em áreas degradadas. Revista Internacional de Ciências, v. 5, n. 2, p. 127-142, 2015.

Disponível em: https://www.researchgate.net/publication/287109728_RESPIRACAO_DO_SOLO_C OMO_BIOINDICADOR_EM_AREAS_DEGRADADAS̄> 10.12957/ric.2015.19581

VERONA, L. A. F; CASALINHO, H.; MASERA, O.; GALVÁN, Y.; CORRÊA, I. et al. Uso de indicadores compostos na análise da sustentabilidade de agroecossistemas de base familiar na região sul do Rio Grande do Sul. Revista Brasileira de Agroecologia, v. 2, n. 2, p. 491-494, 2007. Disponível em: < http://revistas.abaagroecologia.org.br/index.php/cad/article/view/2822>

VEUM, K. S.; SUDDUTH, K. A.; KREMER, R. J.; KITCHEN, N. Sensor data fusion for soil health assessment. Geoderma, 305:53-61, 2017. Disponível em: < https://www.sciencedirect.com/science/article/pii/S0016706116305390> DOI: 10.1016/j.geoderma.2017.05.031 